\title{
Chemical ligation from O-acyl isopeptides via 8- and 11-membered cyclic transition states
}

\author{
Siva S. Panda, ${ }^{a}$ Mohamed Elagawany, ${ }^{\text {a,b }}$ Hadi M. Marwani, ${ }^{\mathrm{c}}$ Eray Çalışkan, ${ }^{\mathrm{a}}$ Mirna EI \\ Khatib, ${ }^{a}$ Alexander Oliferenko, ${ }^{a}$ Khalid A. Alamry, ${ }^{c}$ and Alan R. Katritzky ${ }^{* a, c}$ \\ ${ }^{a}$ Center for Heterocyclic Compounds, Department of Chemistry, \\ University of Florida, Gainesville, FL 32611-7200 (USA) \\ ${ }^{b}$ Department of Organic Chemistry, College of Pharmacy, Misr University for Science and \\ Technology, Al-Motamayez District, P.O. Box: 77, Egypt \\ ${ }^{c}$ Department of Chemistry, King Abdulaziz University, Jeddah, 21589, Saudi Arabia \\ E-mail:Katritzky@chem.ufl.edu
}

DOI: http://dx.doi.org/10.3998/ark.5550190.p008.632

\begin{abstract}
Unprotected $\mathrm{O}$-acylated serine and $\mathrm{O}$-acylated threonine isopeptides have been synthesized, and their conversion to native tripeptides and tetrapeptides by $\mathrm{O}$ - to $\mathrm{N}$-terminus transfer investigated. Ligations involving 8- and 11-membered cyclic transition states are shown experimentally and computationally to be more favorable than intermolecular cross-ligations.
\end{abstract}

Keywords: Ligation, serine, threonine, peptide, benzotriazole

\section{Introduction}

The crucial importance of peptides in biological systems and their potent therapeutic activity ${ }^{1-4}$ has increased the demand for efficient preparative methods. Synthetic access to proteins allows changes to be made in their covalent structure and enables specific labelling of a protein. ${ }^{5-7}$

Merrifield's linear solid-phase peptide synthesis (SPPS) is commonly used in polypeptide synthesis ${ }^{9}$ but, linear SPPS of a large polypeptide can be tedious and costly. Thus, techniques to achieve convergent synthesis from smaller polypeptide fragments are critical in terms of reducing the production costs of peptide therapeutics and the synthesis of proteins. ${ }^{5-7}$

Chemoselective ligations are increasingly the 'key to the success' of protein synthesis. ${ }^{10-13}$ Native chemical ligation (NCL), uniquely joins two unprotected peptide segments chemoselectively and regioselectively to afford a native peptide bond. ${ }^{5,7}$ As reported in 1953 by Wieland et al. ${ }^{8} \mathrm{NCL}$ can join two peptide fragments through a thioester transesterification step followed by an intramolecular rearrangement involving an $\mathrm{S}$ - to $\mathrm{N}$-acyl transfer via a cyclic 
transition state resulting in the native peptide bond. ${ }^{7,8}$ The bifunctional nature of the N-terminal cysteine 1,2-mercaptoamine moiety is responsible for the observed chemoselectivity in NCL. ${ }^{14}$

NCL development as a synthetic tool for building peptides depends on additives to increase ligation rates and yields. Ligations depend on factors such as steric demand, the exogenous thiol reactivity and the nature of the solvent. ${ }^{14-17}$ In addition, the low abundance of cysteine $(1.7 \%$ of the residues in protein sequences) is a major drawback of this methodology since NCL is restricted to Cys residues.

Our focus has been on serine and threonine, each possessing the 1,2-hydroxylamine bifunctionality ${ }^{14}$ (corresponding to the $\mathrm{SH} / \mathrm{NH}_{2}$ in cysteine) thus affording chemoselective ligation by $\mathrm{O}$ - to $\mathrm{N}$ - acyl transfer without the need of cysteine residues. Acylation of the hydroxyl groups of serine and threonine, difficult without epimerization (especially in solidphase synthesis) ${ }^{18,19}$ was recently simplified by a solution-phase entry to enantiopure O-acyl isopeptides using acyl-benzotriazoles. ${ }^{20}$

Our group developed ligations of S-acylated Cys-peptides, ${ }^{21-23}$ and N-acylated Trppeptides. ${ }^{24}$ Recently we demonstrated such classic $\mathrm{O}$ - to $\mathrm{N}$-acyl migration via various transition states in O-acylated serine and tyrosine isopeptides. ${ }^{25,26}$

We now report an experimental and computational study of "traceless" chemical ligation involving O- to N- acyl shift (at Ser and Thr sites) with 8-and 11-membered transition states, involving neither cysteine nor an auxiliary group at the ligation site.

\section{Results and Discussion}

Monoisotripeptides 5a-g were synthesized as starting materials to study the possibility of O- to $\mathrm{N}$-acyl migrations via 8-membered cyclic transition state. Compounds 5a,f were used as starting material for the synthesis of monoisotetrapeptide 9a,b for ligation studies via an 11-membered cyclic transition state.

\section{Preparation of the monoisotripeptides 5a-g}

$N$-(Pg- $\alpha$-Aminoacyl)benzotriazoles 1a-c were coupled with L-Ser-OH 2a/L-Thr-OH $\mathbf{2 b}$ using a previously reported method ${ }^{20}$ to give the corresponding protected dipeptides 3a-e. Dipeptides 3a-e were O-acylated by Cbz-L-Ala-Bt 1b, Boc-L-Phe-Bt 1c, Boc-Gly-Bt 1d or Boc-L-Val-Bt 1e in the presence of diisopropylethylamine (DIPEA) to provide N-protected monoisotripeptides 4a-g (71-86\%), which after deprotection by hydrogenation with $\mathrm{Pd} / \mathrm{C}$ in methanol or $\mathrm{HCl}$ solution in 1,4-dioxane, yielded the free monoisotripeptides 5a-g (Scheme 1, Table 1). Peptides 5a,f were used both directly for ligation studies and also as intermediates to prepare the monoisotetrapeptides $\mathbf{9 a , b}$. 


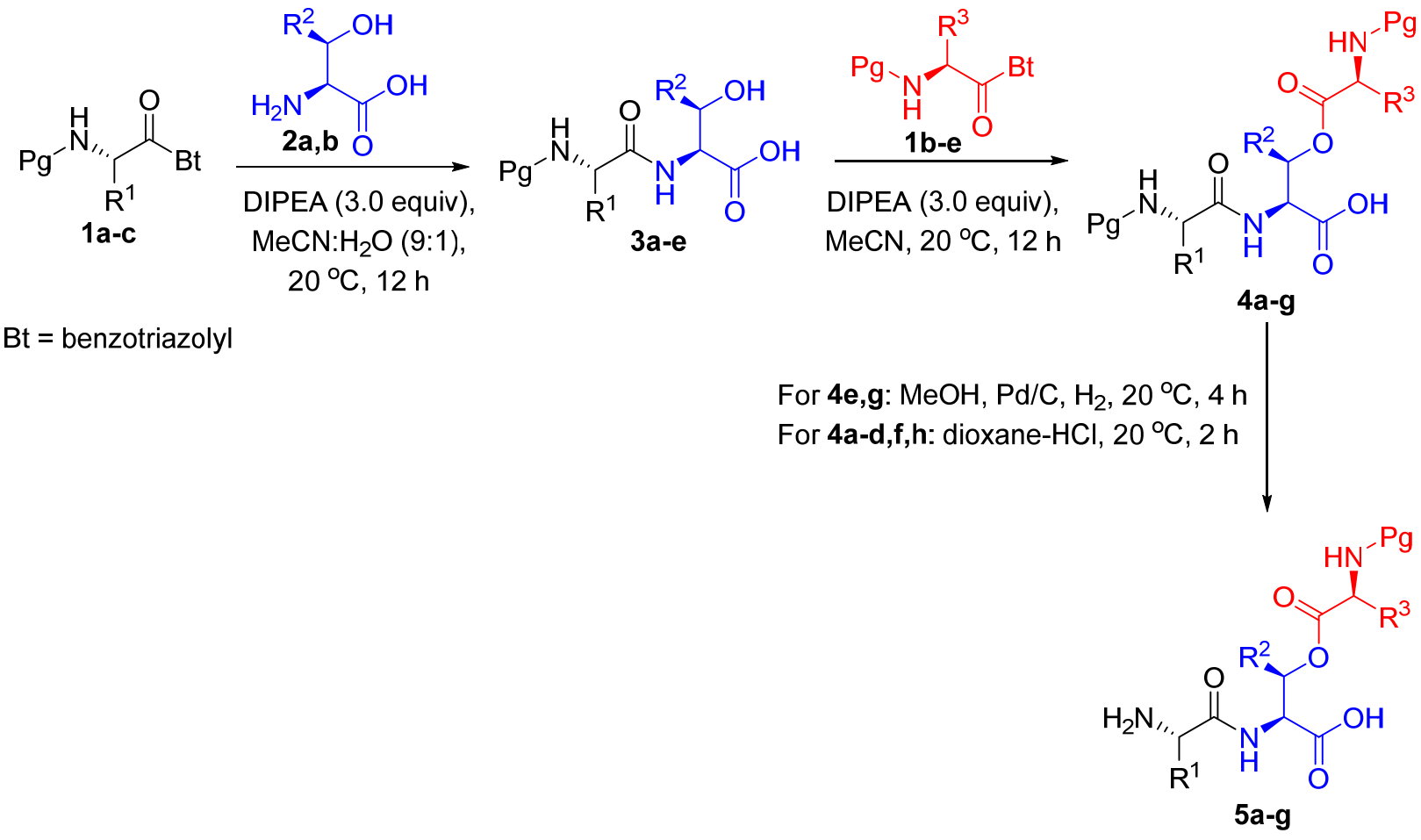

Scheme 1. Preparation of monoisotripeptide 5a-g.

Table 1. Preparation of monoisotripeptides

\begin{tabular}{lccccc}
\hline \multicolumn{1}{c}{ Product 4 } & Yield $(\%)$ & $\mathrm{Mp}\left({ }^{\circ} \mathrm{C}\right)$ & Product 5 & Yield (\%) & $\mathrm{Mp}\left({ }^{\circ} \mathrm{C}\right)$ \\
\hline Cbz-L-Phe-Ser(Boc-Gly)-OH, 4a & 86 & $88-90$ & $\mathbf{5 a}$ & 80 & $170-172$ \\
Cbz-L-Ala-Ser(Boc-L-Phe)-OH, 4b & 73 & $66-68$ & $\mathbf{5 b}$ & 79 & $150-152$ \\
Cbz-L-Ala-Ser(Boc-Gly)-OH, 4c & 75 & $58-60$ & $\mathbf{5 c}$ & 85 & $108-110$ \\
Cbz-L-Ala-Ser(Boc-L-Val)-OH, 4d & 71 & $73-75$ & $\mathbf{5 d}$ & 75 & gum \\
Boc-L-Phe-Ser(Cbz-L-Ala)-OH, 4e & 79 & $72-73$ & $\mathbf{5 e}$ & 95 & $103-104$ \\
Cbz-L-Phe-Thr(Boc-Gly)-OH, 4f & 80 & oil & $\mathbf{5 f}$ & 79 & $148-154$ \\
Boc-L-Phe-Thr(Cbz-L-Ala)-OH, 4g & 83 & $72-74$ & $\mathbf{5 g}$ & 92 & $93-95$ \\
\hline
\end{tabular}

\section{Study of the feasibility of $\mathrm{O} \rightarrow \mathrm{N}$ acyl migrations via 8-membered cyclic transition state}

The O- to $\mathrm{N}$-acyl migration for monoisotripeptides 5a-g were studied under microwave irradiation in piperidine-DMF $(20 \mathrm{v} / \mathrm{v} \%), 50{ }^{\circ} \mathrm{C}, 50 \mathrm{~W}, 1 \mathrm{~h}$ to generate native peptides. Anhydrous conditions were chosen to minimize ester hydrolysis. HPLC-MS indicated the formation the ligated products $\mathbf{6 a}-\mathbf{g}$ along with the bis-acylated product $7 \mathbf{a}-\mathbf{g}$. The results show that the nature of amino acids used has a profound effect on the yield of ligated product (Scheme 2, Table 2).

The desired O- to N-acyl transfer migration was only successful for of $\mathbf{5 a}-\mathbf{c}$, e. The retention times and fragmentation patterns of starting material and ligated products were also studied in 
control experiments (HPLC-MS of pure monoisotripeptide). Thus HPLC-MS, via (-)ESIMS/MS, confirmed that compounds 5a-c, e have different fragmentation patterns than 6a-c,e proving the formation of the intramolecular ligated product.

The effect of $\mathrm{O}$ - to $\mathrm{N}$-acyl transfer rearrangement using serine versus threonine for the same amino acid sequence was noted, with a $57 \%$ yield of native peptide $\mathbf{6 a}$ (L-Ser) but none from $\mathbf{6 f}$ (L-Thr). Microwave irradiation for $18 \mathrm{~h}$ at $50{ }^{\circ} \mathrm{C}, 50 \mathrm{~W}$ in piperidine-DMF $(20 \mathrm{v} / \mathrm{v} \%)$ did not alter $\mathbf{5 g}$ significantly and gave no $\mathbf{6 g}$. The addition of salt, such as $\mathrm{NaCl}$, and microwave irradiation $\mathbf{5 g}$ in piperidine-DMF $20 \mathrm{v} / \mathrm{v} \%, 70{ }^{\circ} \mathrm{C}, 50 \mathrm{~W}$ for $10 \mathrm{~h}$ ineffective in the formation of 6g.

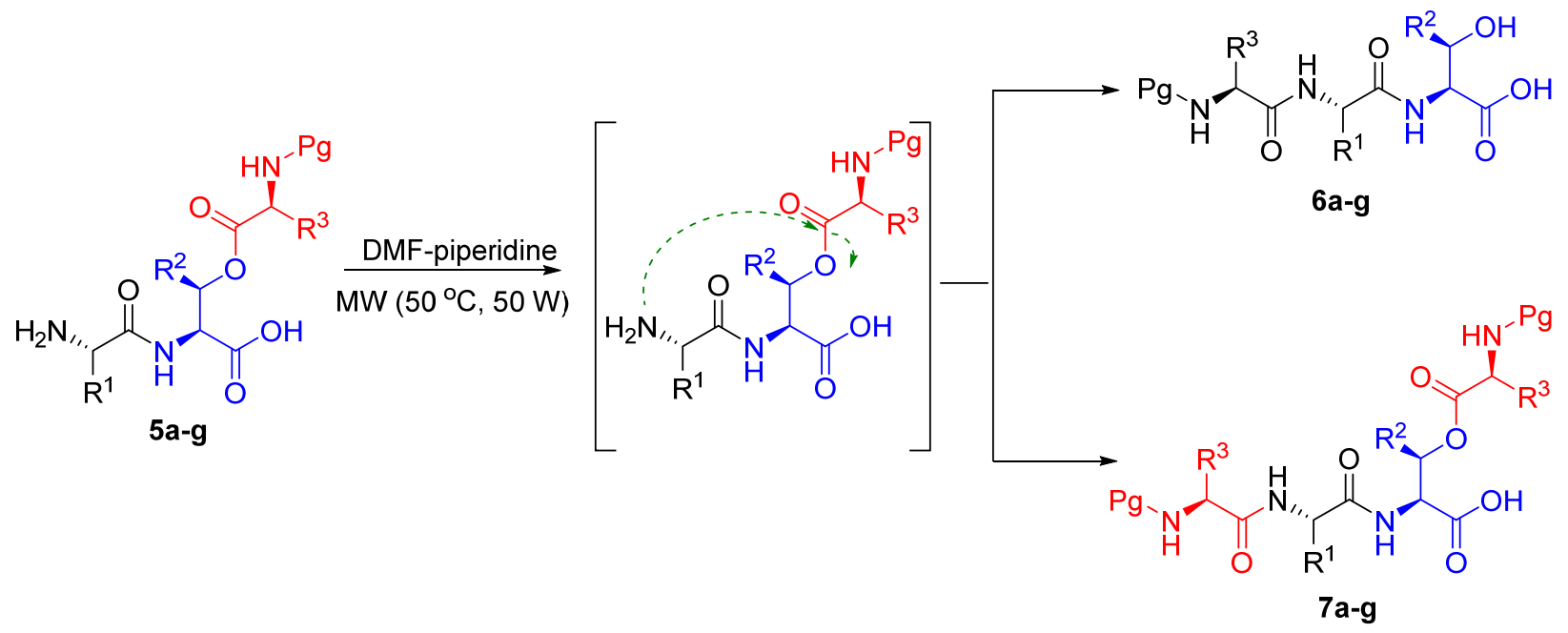

Scheme 2. Acyl migration of O-acyl isotripeptides $\mathbf{5 a}-\mathbf{g}$.

Table 2. Chemical ligation of O-acyl isotripeptides $\mathbf{5 a}-\mathbf{g}$

\begin{tabular}{llll}
\hline Entry & Reactant $\mathbf{5}$ & ${\text { Ligated product } \mathbf{6}(\%)^{\mathrm{a}}}^{\mathrm{a}}$ & Bis-acylated 7 (\%) $^{\mathrm{a}}$ \\
\hline 1 & L-Phe-Ser(Boc-Gly)-OH, 5a & 57 & 0 \\
2 & L-Ala-Ser(Boc-L-Phe)-OH, 5b & 78 & 0 \\
3 & L-Ala-Ser(Boc-Gly)-OH, 5c & 23 & 7 \\
4 & L-Ala-Ser(Boc-L-Val)-OH, 5d & 0 & 4 \\
5 & L-Phe-Ser(Cbz-L-Ala)-OH, 5e & 8 & 1 \\
6 & L-Phe-Thr(Boc-Gly)-OH, 5f & 0 & 0.5 \\
7 & L-Phe-Thr(Cbz-L-Ala)-OH, 5g & 0 & 0 \\
\hline
\end{tabular}

${ }^{\text {a }}$ Semiquantitative determination HPLC-MS. The area of ion-peak resulting from the sum of the intensities of the $[\mathrm{M}+\mathrm{H}]^{+}$and $[\mathrm{M}+\mathrm{Na}]^{+}$ions for each compound was integrated (corrected for starting material). 


\section{Computational rationalization}

Conformational preorganization has been shown ${ }^{27}$ to be important for internal ligation. In previous publications ${ }^{20,23}$ we have demonstrated this principle and shown that sterically hindered and thus poorly preorganized isopeptides gave lower yields.

The reactivity of O-acyl isopeptides $\mathbf{5 a - g}$ also varies with the protecting group and the nature of amino acid adjacent to the protecting group. To rationalize the reactivity patterns of $\mathbf{5 a}-\mathbf{g}$, a previously developed computational protocol $^{27}$ were applied, which included a full conformational search and virtual screening based on a purposefully defined scoring function. This function $\mathbf{b}(\mathrm{N}-\mathrm{C})$ is the geometrical distance between the amine nucleophile and the target ester carbon atom. Conformational searches were performed using the MMX force field (as implemented in PCModel v.9.3 software). The $\mathbf{b}(\mathrm{N}-\mathrm{C})$ values are given in Table 3 for all the isopeptides. Generally the scoring function values are in qualitative but not perfect agreement with the yields. This can be explained by the presence of additional degrees of freedom. By analyzing the preorganized structures we found that the nucleophilic attack can be attenuated by hydrogen bond contact between one of the amino group protons and the oxygen atom belonging to the target carbonyl group. These contacts are characterized by bond separations $\mathbf{b}(\mathrm{H}-\mathrm{O})$, which are also listed in Table 3. There is a clear relationship between the yield and the hydrogen bond strength characterized by $\mathbf{b}(\mathrm{H}-\mathrm{O})$ : the shorter the $\mathbf{b}(\mathrm{H}-\mathrm{O})$ the lower the yield. The preorganized structures of $\mathbf{5 b}$ and $\mathbf{5 f}$ are shown in Figure 1: structure $\mathbf{5 f}$ forms a distinct hydrogen bond contact (designated with a dashed line) both in terms of $\mathbf{b}(\mathrm{H}-\mathrm{O})$ and the N-H-O angle, whereas $\mathbf{5 b}$ is definitely not in a hydrogen bond configuration, since the $\mathrm{NH}$ bonds face away from the acceptor atom (Figure 1).

The ratio of $\mathbf{b}(\mathrm{N}-\mathrm{C}): \mathbf{b}(\mathrm{H}-\mathrm{O})$ gives an important measure of conformational preorganization for binding, as the best performing compounds $\mathbf{5 a}-\mathbf{c}$ have lower $\mathbf{b}(\mathrm{N}-\mathrm{C}) / \mathbf{b}(\mathrm{H}-\mathrm{O})$ values. This $\mathrm{NH} . . \mathrm{O}=\mathrm{C}$ is not the only contact that can alter the reactivity: the optimized isopeptide structures disclose additional hydrogen bond contacts capable of locking the structure in an unfavorable conformation. Such conformationally locked structures were found for $\mathbf{5 c}-\mathbf{g}$. The locking hydrogen bonds were characterized not only by bond separations, but also by the H-A...D angles (where A and D stand for acceptor and donor, respectively) are listed in Table 3. Table 3 suggests that hydrogen bond contacts are particularly strong in structures $\mathbf{5 c , d , f . ~ A p p a r e n t l y , ~}$ such hydrogen bond may be supportive, as, for example, in $\mathbf{5 c}$, which locks the structure in a favorable conformation, but this is not always the case, as evident from the zero yields found with $\mathbf{5 d}$ and $\mathbf{5 f}$.

Replacement of Ser by Thr clearly reduces reactivity. This can be deduced by comparing the yields of the target product $\mathbf{6 a -} \mathbf{g}$ in $\mathbf{5 a}$ against $\mathbf{5 f}(57 \%$ vs. 0$)$ and $\mathbf{5 e}$ against $\mathbf{5 g}(8 \%$ vs. 0$)$. In conformational terms, it follows that the Thr methyl group (absent in Ser) drives the N-terminus slightly farther away from the target carbonyl, which is confirmed by larger $\mathbf{b}(\mathrm{N}-\mathrm{C})$ values in $\mathbf{5 f}, \mathbf{g}$ compared with $\mathbf{5 a}$ and $\mathbf{5 e}$, respectively.

Evidently, the preorganization of the starting O-acyl isotripeptides is an important factor in 
the success of intramolecular long range 8-membered transition acyl transfers.
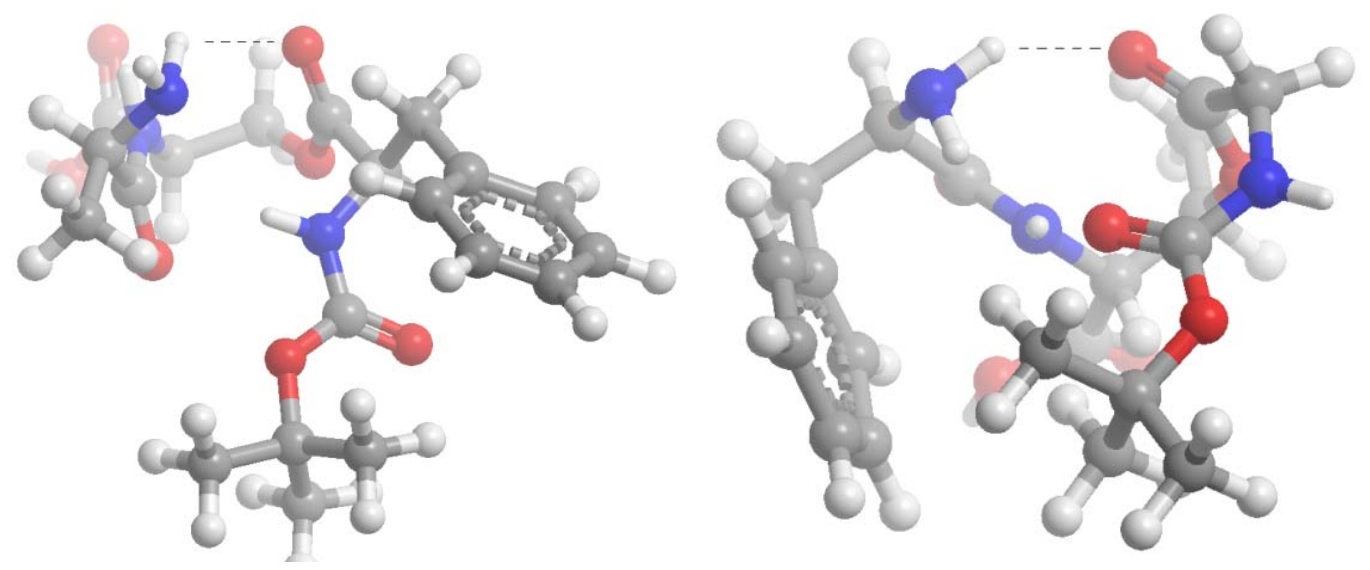

Figure 1. Putative $\mathrm{NH} . . \mathrm{O}=\mathrm{C}$ hydrogen bond contact in preorganized structure $\mathbf{5 f}$ (right); no hydrogen bond in $\mathbf{5 b}$ (left).

Table 3. Structural characteristics of preorganized conformers $\mathbf{5 a}-\mathbf{g}$

\begin{tabular}{|c|c|c|c|c|c|c|c|c|}
\hline Structure 5 & $\begin{array}{c}\mathrm{b}(\mathrm{N}-\mathrm{C}) \\
(\AA)\end{array}$ & $\begin{array}{c}\mathrm{b}(\mathrm{H}-\mathrm{O}) \\
(\AA)\end{array}$ & Ratio & $\begin{array}{c}\text { Yield } 6 \\
(\%)\end{array}$ & Acceptor & Donor & $\begin{array}{c}\mathrm{b}(\mathrm{D}-\mathrm{A}) \\
(\AA)\end{array}$ & $\begin{array}{l}\angle \mathrm{HAD} \\
\text { (deg.) }\end{array}$ \\
\hline $5 \mathbf{a}$ & 3.53 & 2.38 & 1.48 & 57 & - & - & - & - \\
\hline $5 b$ & 3.48 & 2.47 & 1.41 & 78 & - & - & - & - \\
\hline $5 c$ & 3.34 & 2.33 & 1.43 & 23 & Ala N & Boc NH & 1.81 & 174 \\
\hline $5 d$ & 3.26 & 2.17 & 1.50 & - & Ala N & Boc NH & 1.86 & 162 \\
\hline $5 e$ & 3.85 & 2.21 & 1.74 & 8 & Phe O & $\mathrm{Cbz} \mathrm{NH}$ & 2.06 & 148 \\
\hline $5 f$ & 3.75 & 2.06 & 1.82 & - & Boc $\mathrm{O}$ & Thr NH & 1.98 & 156 \\
\hline $5 g$ & 4.25 & 2.80 & 1.52 & - & $\mathrm{CbzO}$ & Phe NH & 2.05 & 135 \\
\hline
\end{tabular}

\section{Preparation of the $\mathbf{O}$-acyl isotetrapeptides $9 \mathbf{a}, \mathbf{b}$}

Cbz protected monoisotetrapeptides 9a,b were synthesized in solution phase, by coupling the benzotriazolides of $\mathrm{Cbz}$ protected Glycine $\mathbf{1 f}$ with the unprotected monoisotripeptides 5a,f at 20 ${ }^{\circ} \mathrm{C}$ in $75-85 \%$ yield. The protecting groups of $\mathbf{8 a}, \mathbf{b}$ were removed by stirring each with $\mathrm{Pd} / \mathrm{C}$ in methanol in hydrogen atmosphere for $1 \mathrm{~h}$ to afford the unprotected monoisotetrapeptides $9 \mathbf{a , b}$ (Scheme 3). Compounds 8a,b and 9a,b were fully characterized by ${ }^{1} \mathrm{H},{ }^{13} \mathrm{C}$ NMR analysis. 

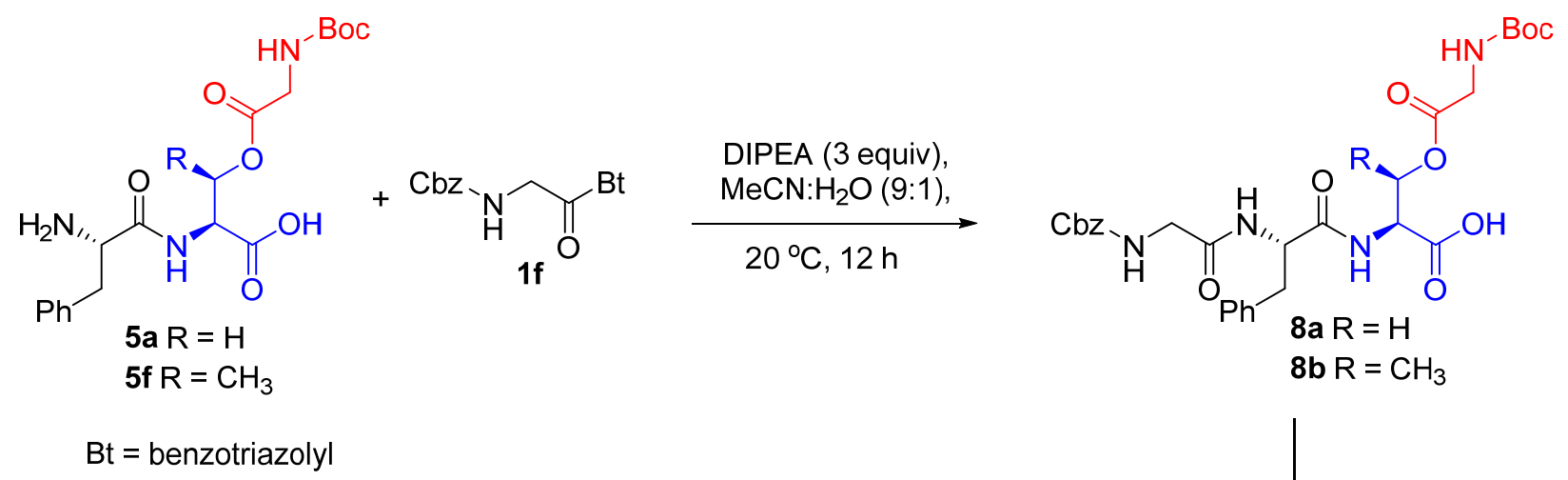

$\mathrm{Bt}=$ benzotriazolyl

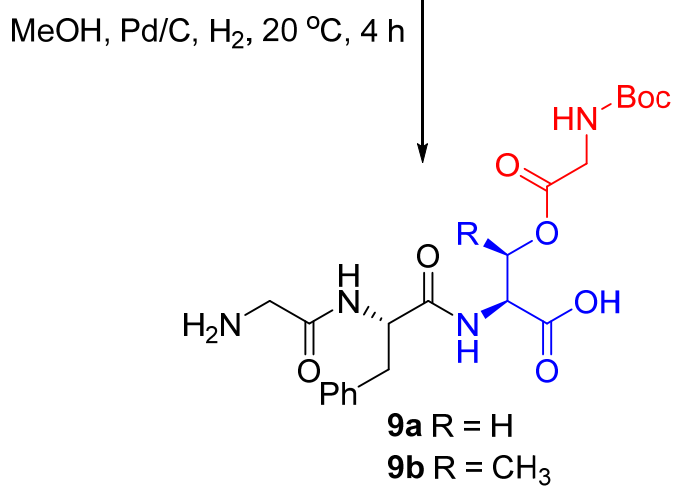

Scheme 3. Synthesis of O-acyl isotetrapeptides 9a,b.

\section{Study of the feasibility of $\mathrm{O} \rightarrow \mathrm{N}$ acyl migrations via an 11-membered cyclic transition state} Intermediates 9a,b underwent ligation (Scheme 4, Table 4) under basic conditions (piperidine 20 $\mathrm{v} / \mathrm{v} \%$ in DMF, MW $50{ }^{\circ} \mathrm{C}, 50 \mathrm{~W}, 1 \mathrm{~h}$ for $9 \mathrm{a}$ and $1 \mathrm{~h}, 3 \mathrm{~h}$, and $6 \mathrm{~h}$ for $9 \mathrm{~b}$ ). Unlike the challenges of steric hindrance and poor organization for binding eight-membered cyclic transition state, the expanded eleven transition state afforded $\mathrm{O}$ - to $\mathrm{N}$-acyl transfer to give the desired native peptide 10a. However, threonine remained a challenge in both eight and eleven ring transition states. HPLC-MS showed the formation of the expected intramolecular ligated products 10a. The retention times and fragmentation patterns of 9a was studied by control experiments (HPLC-MS of pure 9a). Different fragmentation patterns in HPLC-MS, via (-)ESI-MS/MS, were found for 9a and 10a, each of MW 466. In addition, product 10a was isolated and its structure further confirmed by HRMS.

The ligation of 9a (eleven transition state) under aqueous conditions $(\mathrm{pH} 7.6,1 \mathrm{M}$ buffer strength, MW $50{ }^{\circ} \mathrm{C}, 50 \mathrm{~W}, 1 \mathrm{~h}$ ). HPLC-MS gave a small amount of the corresponding ligated product 10a, together with a major peak having MW 366 corresponding to the removal of the Boc-group either from $9 \mathbf{a}$ or from the ligated product 10a. 


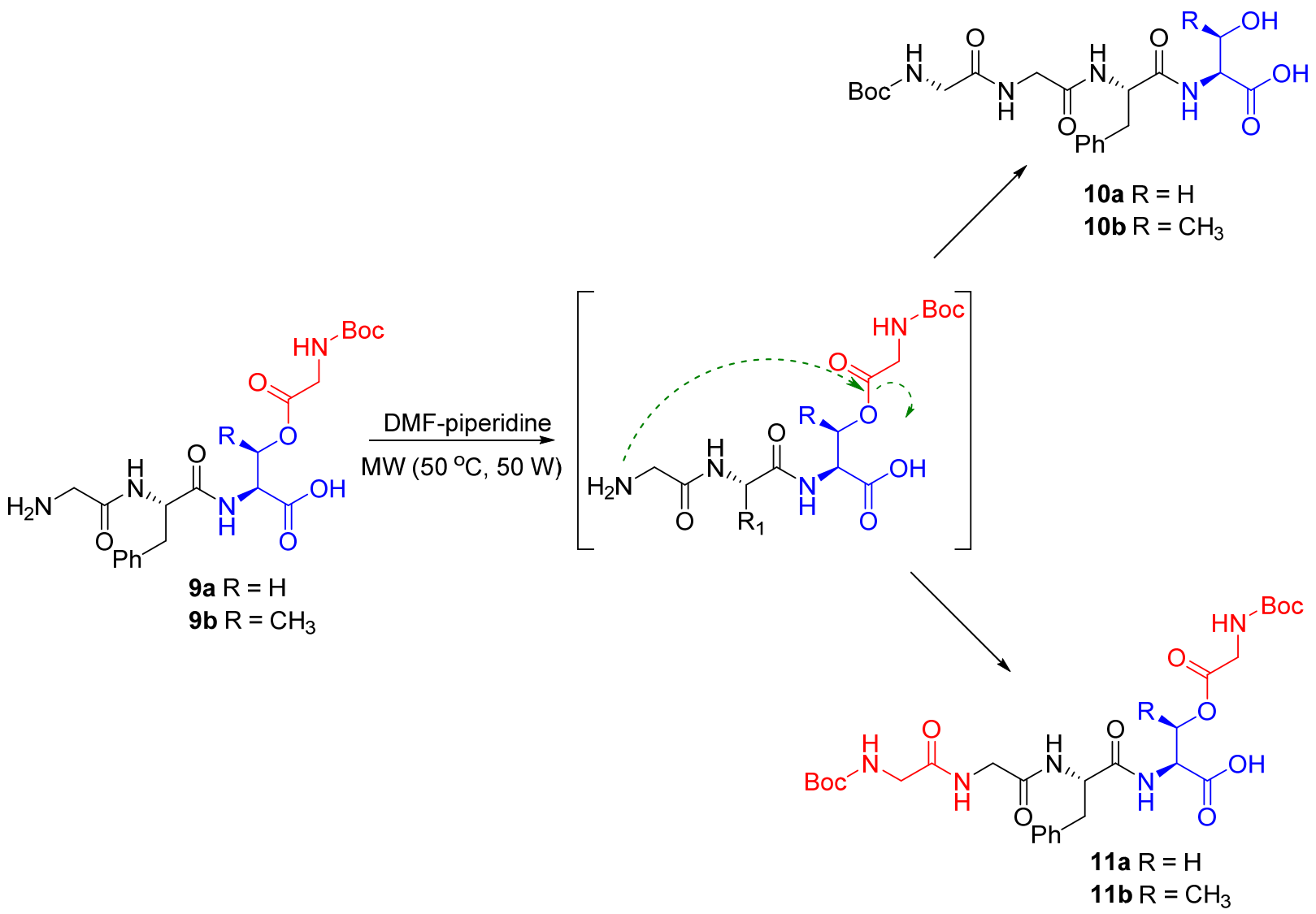

Scheme 4. Acyl migration of O-acyl isotripeptides 9a,b.

Table 4. Chemical ligation of O-acyl isotetrapeptides 9a,b

\begin{tabular}{llll}
\hline Entry & Reactant 9 & Ligated product 10 (\%) $^{\mathrm{a}}$ & Bis-acylated 11 (\%) $^{\mathrm{a}}$ \\
\hline 1 & Gly-L-Phe-L-Ser(Boc-Gly)-OH, 9a & 99 & 0 \\
2 & Gly-L-Phe-Thr(Boc-Gly)-OH, 9b & 0 & 0 \\
\hline
\end{tabular}

${ }^{a}$ Semiquantitative determination HPLC-MS. The area of ion-peak resulting from the sum of the intensities of the $[\mathrm{M}+\mathrm{H}]^{+}$and $[\mathrm{M}+\mathrm{Na}]^{+}$ions for each compound was integrated (corrected for starting material).

\section{Conclusions}

In summary, the chemical ligation of peptides affording O-acyl isopeptides occurs successfully without the use of cysteine or an auxiliary group. However, conformation preorganization of such peptides is critical for successful O- to $\mathrm{N}$-acyl transfer to afford the corresponding native peptides. 


\section{Experimental Section}

General. Melting points were determined on a capillary point apparatus equipped with a digital thermometer and are uncorrected. NMR spectra were recorded with TMS for ${ }^{1} \mathrm{H}(300 \mathrm{MHz})$ and ${ }^{13} \mathrm{C}(75 \mathrm{MHz})$ as an internal reference. Starting materials are available commercially and used without further purification. Reaction progress was monitored by thin-layer chromatography (TLC) and visualized by UV light. Elemental analyses were performed on a Carlo Erba EA 1108 instrument. HPLC-MS analyses were performed on reverse phase gradient Phenomenex Synergi Hydro-RP (C18): $(2 \times 150 \mathrm{~mm}$; $4 \mathrm{um})+\mathrm{C} 18$ guard column $(2 \times 4 \mathrm{~mm})$, wavelength $=254 \mathrm{~nm}$; flow rate $0.2 \mathrm{~mL} / \mathrm{min}$; and mass spectrometry was done with electro spray ionization (ESI).

General procedure for the preparation of dipeptides (3a-e). $\quad N-(\mathrm{Pg}-\alpha-$ Aminoacyl)benzotriazoles $(1.0 \mathrm{mmol})$ in $\mathrm{MeCN}(5 \mathrm{~mL})$ was added dropwise to a solution of free amino acid (1.5-2.0 equiv) and DIPEA (3.0 equiv) in $\mathrm{MeCN}$ (sometimes $\mathrm{MeCN} / \mathrm{H}_{2} \mathrm{O}$ 9:1, 15 $\mathrm{mL})$ at the room temperature and stirred until all the $\mathrm{N}$-(Pg- $\alpha$-aminoacyl)benzotriazoles were consumed $(12 \mathrm{~h}) . \mathrm{MeCN}$ was evaporated and the residue dissolved in EtOAc $(50 \mathrm{~mL})$ and washed with $3 \mathrm{~N} \mathrm{HCl}(5 \times 50 \mathrm{~mL})$. The organic portion was dried over anhyd. $\mathrm{Na}_{2} \mathrm{SO}_{4}$, filtered and concentrated to give the desired peptide fragment. No further purification was required in all cases.

((Benzyloxy)carbonyl)-L-phenylalanyl-L-serine (Cbz-L-Phe-L-Ser-OH, 3a). White solid (85\%); mp 156-157 ${ }^{\circ} \mathrm{C} ;{ }^{1} \mathrm{H}$ NMR $\left(\mathrm{CD}_{3} \mathrm{OD}\right) \delta 8.16(\mathrm{~d}, J 7.8 \mathrm{~Hz}, 1 \mathrm{H}), 7.38-7.20(\mathrm{~m}, 10 \mathrm{H}), 5.05-$ $4.80(\mathrm{~m}, 2 \mathrm{H}), 4.52-4.42(\mathrm{~m}, 2 \mathrm{H}), 3.95-3.80(\mathrm{~m}, 2 \mathrm{H}), 3.23-3.16(\mathrm{~m}, 1 \mathrm{H}), 2.90-2.81(\mathrm{~m}, 1 \mathrm{H}) ;{ }^{13} \mathrm{C}$ NMR $\left(\mathrm{CD}_{3} \mathrm{OD}\right) \delta 174.3,173.2,158.4,138.7,138.2,130.5,129.6,129.0,128.8,127.8,67.7$, 63.0, 57.9, 56.2, 39.3. Anal. Calcd for $\mathrm{C}_{20} \mathrm{H}_{22} \mathrm{~N}_{2} \mathrm{O}_{6}$ : C, 62.17; H, 5.74; N, 7.25; Found: C, 62.47; $\mathrm{H}, 5.82 ; \mathrm{N}, 7.21$.

((Benzyloxy)carbonyl)-L-alanyl-L-serine (Cbz-L-Ala-L-Ser-OH, 3b). White solid (81\%); 195$197{ }^{\circ} \mathrm{C}$; (lit. ${ }^{28} \mathrm{mp} 192-194{ }^{\circ} \mathrm{C}$ ); ${ }^{1} \mathrm{H}$ NMR (DMSO- $\left.d_{6}\right) \delta 7.99(\mathrm{~d}, J 7.8 \mathrm{~Hz}, 1 \mathrm{H}), 7.46(\mathrm{~d}, J 7.8 \mathrm{~Hz}$, $1 \mathrm{H}), 7.36-7.29(\mathrm{~m}, 5 \mathrm{H}), 5.02(\mathrm{~s}, 2 \mathrm{H}), 4.29-4.23(\mathrm{~m}, 1 \mathrm{H}), 4.17-4.10(\mathrm{~m}, 1 \mathrm{H}), 3.72(\mathrm{dd}, J 11,5$ $\mathrm{Hz}, 1 \mathrm{H}) 3.62(\mathrm{dd}, J 11,4 \mathrm{~Hz}, 1 \mathrm{H}), 1.21(\mathrm{~d}, J 7.1 \mathrm{~Hz}, 3 \mathrm{H}) ;{ }^{13} \mathrm{C}$ NMR (DMSO- $\left.d_{6}\right) \delta 172.5,171.9$, 155.6, 137.0, 128.3, 127.8, 127.7, 65.4, 61.3, 54.6, 49.8, 18.3 .

(tert-Butoxycarbonyl)-L-phenylalanyl-L-serine (Boc-L-Phe-L-Ser-OH, 3c). White solid (80\%); mp 63-65 ${ }^{\circ} \mathrm{C} ;{ }^{1} \mathrm{H}$ NMR $\left(\mathrm{CDCl}_{3}\right) \delta$ 7.47-7.28 (m, 5H), $5.02(\mathrm{br} \mathrm{s}, 2 \mathrm{H}), 4.29-4.22(\mathrm{~m}, 1 \mathrm{H})$, 4.17-4.10 (m, 1H), 3.76-3.71 (m, 1H), 3.66-3.59 (m, 1H), 1.23-1.20 (m, 9H); ${ }^{13} \mathrm{C}$ NMR $\left(\mathrm{CDCl}_{3}\right) \delta 172.7,172.1,156.1,136.3,129.4,128.5,126.9,80.8,62.6,55.5,54.7,38.7,28.2$, 28.0. Anal. Calcd for $\mathrm{C}_{17} \mathrm{H}_{24} \mathrm{~N}_{2} \mathrm{O}_{6}$ : C, 57.94; H, 6.86; N, 7.95; Found: C, 57.83; H, 7.34; N, 7.47.

((Benzyloxy)carbonyl)-L-phenylalanyl-L-threonine (Cbz-L-Phe-L-Thr-OH, 3d). White solid (89\%); mp 51-53 ${ }^{\circ} \mathrm{C} ;{ }^{1} \mathrm{H}$ NMR $\left(\mathrm{CD}_{3} \mathrm{OD}\right) \delta 7.40-7.20(\mathrm{~m}, 10 \mathrm{H}), 4.55-4.46(\mathrm{~m}, 2 \mathrm{H}), 4.34(\mathrm{br} \mathrm{s}$, $1 \mathrm{H}), 4.10-4.06(\mathrm{~m}, 1 \mathrm{H}), 3.30-3.18(\mathrm{~m}, 1 \mathrm{H}), 2.95-2.88(\mathrm{~m}, 1 \mathrm{H}), 1.96(\mathrm{br} \mathrm{s}, 1 \mathrm{H}), 1.20-1.15(\mathrm{~m}$, $3 \mathrm{H}) ;{ }^{13} \mathrm{C}$ NMR $\left(\mathrm{CD}_{3} \mathrm{OD}\right) \delta 174.1,173.1,157.8,138.1,137.7,130.1,129.0,128.5,128.3,127.3$, 
68.3, 67.2, 58.7, 57.4, 38.7, 20.2. HRMS $m / z$ for $\mathrm{C}_{21} \mathrm{H}_{24} \mathrm{~N}_{2} \mathrm{O}_{6}[\mathrm{M}+\mathrm{Na}]^{+}$calcd. 423.1527, found 423.1514 .

(tert-Butoxycarbonyl)-L-phenylalanyl-L-threonine (Boc-L-Phe-L-Thr-OH, 3e). Yellow gel ${ }^{29}$ (78\%); ${ }^{1} \mathrm{H}$ NMR $\left(\mathrm{CDCl}_{3}\right) \delta 7.57(\mathrm{~d}, J 7.9 \mathrm{~Hz}, 1 \mathrm{H}), 7.25-7.19(\mathrm{~m}, 5 \mathrm{H}), 5.70(\mathrm{~d}, J 7.5 \mathrm{~Hz}, 1 \mathrm{H})$, 4.75-4.25 (m, 3H), 3.25-3.08 (m, 1H), 2.99-2.81 (m, 1H), $1.33(\mathrm{~s}, 9 \mathrm{H}), 1.17(\mathrm{~d}, J 5.8 \mathrm{~Hz}, 3 \mathrm{H})$; ${ }^{13} \mathrm{C} \mathrm{NMR}\left(\mathrm{CDCl}_{3}\right) \delta 173.4,173.1,156.1,136.5,129.5,128.5,126.9,80.5,68.1,57.6,28.3,19.5$.

General procedure for the preparation of $\mathbf{O}$-acyl isopeptides $4 \mathbf{a}-\mathbf{g}$. Compound $\mathbf{3}$ (1.0 mmol) was added to a solution of $N$-(Pg- $\alpha$-aminoacyl)benzotriazoles $(1.0 \mathrm{mmol})(1.0 \mathrm{mmol})$ and DIPEA $(3.0 \mathrm{mmol})$ in $\mathrm{MeCN}(20 \mathrm{~mL})$ at room temperature and stirred for $12 \mathrm{~h}$. MeCN was evaporated and the residue dissolved in EtOAc $(50 \mathrm{~mL})$ and washed with $2 \mathrm{~N} \mathrm{HCl}(3 \times 50 \mathrm{~mL})$. The organic portion was dried over anhyd. $\mathrm{Na}_{2} \mathrm{SO}_{4}$, filtered and concentrated to give $4 \mathbf{a}-\mathbf{g}$.

$\mathrm{N}$-(((Benzyloxy)carbonyl)-L-phenylalanyl)-O-((tert-butoxycarbonyl)glycyl)-L-serine (Cbz-LPhe-L-Ser(Boc-Gly)-OH, 4a). White solid. (86\%); mp 86-88 ${ }^{\circ} \mathrm{C} ;{ }^{1} \mathrm{H}$ NMR $\left(\mathrm{CD}_{3} \mathrm{OD}\right) \delta 8.35(\mathrm{~d}$, $J$ 8.1Hz, 1H), 7.33-7.17 (m, $10 \mathrm{H}), 5.01-4.98(\mathrm{~m}, 2 \mathrm{H}), 4.77-4.73(\mathrm{~m}, 1 \mathrm{H}), 4.57$ (dd, $J 11.4,3.6$ $\mathrm{Hz}, 1 \mathrm{H}), 4.48-4.36(\mathrm{~m}, 2 \mathrm{H}), 3.78$ (s, 2H), 3.18 (dd, J 13.8, 4.5 Hz, 1H), 2.89-2.80 (m, 1H), 1.42 $(\mathrm{s}, 9 \mathrm{H}) ;{ }^{13} \mathrm{C} \mathrm{NMR}\left(\mathrm{CD}_{3} \mathrm{OD}\right) \delta 174.3,171.8,158.6,158.3,138.6,138.2,130.5,129.5,129.0$, 128.8, 127.8, 80.9, 67.7, 65.0, 57.8, 53.0, 43.0, 39.2, 28.9. Anal. Calcd for $\mathrm{C}_{27} \mathrm{H}_{33} \mathrm{~N}_{3} \mathrm{O}_{9}$ : C, 59.66; H, 6.12; N, 7.73; Found C, 59.62; H, 6.13; N, 6.96.

$\mathrm{N}$-(((Benzyloxy)carbonyl)-L-alanyl)-O-((tert-butoxycarbonyl)-L-phenylalanyl)-L-serine (Cbz-L-Ala-L-Ser(Boc-L-Phe)-OH, 4b). White solid (73\%); mp 66-68 ${ }^{\circ} \mathrm{C} ;{ }^{1} \mathrm{H}$ NMR $\left(\mathrm{CDCl}_{3}\right) \delta$ 7.89 (br s, 2H), 7.40-7.16 (m, 10H), 5.66-5.56 (m, 1H), 5.21-5.10 (m, 2H), 5.08-4.98 (m, 2H), $4.81-4.23(\mathrm{~m}, 3 \mathrm{H}), 3.22-2.88(\mathrm{~m}, 2 \mathrm{H}), 1.40-1.28(\mathrm{~m}, 9 \mathrm{H}) ;{ }^{13} \mathrm{C} \mathrm{NMR}\left(\mathrm{CDCl}_{3}\right) \delta 172.4,172.1$, 171.3, 156.4, 155.7, 136.7, 135.8, 129.3, 128. 5, 128.2, 128.1, 126.7, 80.3, 67.3, 63.7, 56.8, 51.8, 48.9, 38.3, 28.2, 17.5. Anal. Calcd for $\mathrm{C}_{28} \mathrm{H}_{35} \mathrm{~N}_{3} \mathrm{O}_{9}$ : C, 60.31; H, 6.33; N, 7.54; Found C, 60.34; H, 6.74; N, 7.37.

$\mathrm{N}$-(((Benzyloxy)carbonyl)-L-alanyl)-O-((tert-butoxycarbonyl)glycyl)-L-serine (Cbz-L-Ala-LSer(Boc-Gly)-OH, 4c). White solid (75\%); mp 58-60 ${ }^{\circ} \mathrm{C} ;{ }^{1} \mathrm{H}$ NMR (DMSO- $\left.d_{6}\right) \delta 8.24$ (d, $J 7.2$ $\mathrm{Hz}, 1 \mathrm{H}), 7.45$ (d, J 7.5 Hz, 1H), 7.36-7.30 (m, 5H), 7.21 (t, J $12.0 \mathrm{~Hz}, 1 \mathrm{H}), 5.01(\mathrm{~s}, 2 \mathrm{H}), 4.5$ (s, $1 \mathrm{H}), 4.32-4.24(\mathrm{~m}, 2 \mathrm{H}), 4.13-4.08(\mathrm{~m}, 1 \mathrm{H}), 3.68(\mathrm{t}, J 10.5 \mathrm{~Hz}, 2 \mathrm{H}), 1.45-1.23(\mathrm{~m}, 9 \mathrm{H}), 1.20(\mathrm{~d}$, $J 7.2 \mathrm{~Hz}, 3 \mathrm{H}) ;{ }^{13} \mathrm{C}$ NMR (DMSO-d $) \delta 172.7,170.5,170.2,155.8,155.6,137.0,128.3,127.8$, 78.3, 65.4, 63.5, 50.9, 49.8, 41.7, 28.2, 18.2. Anal. Calcd for $\mathrm{C}_{21} \mathrm{H}_{29} \mathrm{~N}_{3} \mathrm{O}_{9}: \mathrm{C}, 53.96 ; \mathrm{H}, 6.25 ; \mathrm{N}$, 8.99; Found C, 53.28; H, 6.49; N, 7.81 .

$\mathrm{N}$-(((Benzyloxy)carbonyl)-L-alanyl)-O-((tert-butoxycarbonyl)-L-valyl)-L-serine (Cbz-L-AlaL-Ser(Boc-L-Val)-OH, 4d). White solid (71\%); mp 73-75 ${ }^{\circ} \mathrm{C} ;{ }^{1} \mathrm{H}$ NMR $\left(\mathrm{CDCl}_{3}\right) \delta 7.33-7.28$ $(\mathrm{m}, 5 \mathrm{H}), 5.24(\mathrm{~d}, 1 \mathrm{H}), 5.15-5.03(\mathrm{~m}, 2 \mathrm{H}), 4.84$ (br s, 1H), 4.54 (br s, 1H), 4.42-4.07 (m, 2H), 2.13-2.04 (m, 1H), 1.43-1.38 (m, 12H), $0.89(\mathrm{dd}, J 16.4,6.6 \mathrm{~Hz}, 6 \mathrm{H}) ;{ }^{13} \mathrm{C} \mathrm{NMR}\left(\mathrm{CDCl}_{3}\right) \delta$ $173.3,172.3,171.5,156.3,136.3,128.6,128.3,128.2,80.5,67.2,63.8,58.9,51.9,50.7,30.9$, 28.4, 18.8, 17.8. Anal. Calcd for $\mathrm{C}_{24} \mathrm{H}_{35} \mathrm{~N}_{3} \mathrm{O}_{9}$ : C, 56.57; H, 6.92; N, 8.25; Found C, 56.92; H, $7.35 ; \mathrm{N}, 8.02$. 
O-(((Benzyloxy)carbonyl)-L-alanyl)- $\mathrm{N}$-((tert-butoxycarbonyl)-L-phenylalanyl)-L-serine (Boc-L-Phe-L-Ser(Cbz-L-Ala)-OH, 4e). White solid. (79\%); mp 72-73 ${ }^{\circ} \mathrm{C} ;{ }^{1} \mathrm{H}$ NMR $\left(\mathrm{CDCl}_{3}\right) \delta$ 7.85 (br s, 2H), 7.36-7.16 (m, 10H), 5.65 (br s, 1H), 5.21-4.98 (m, 2H), 4.80-4.69 (m, 2H), 4.53-4.23 (m, 3H), 3.24-2.88 (m, 2H), 1.39-1.28 (m, 12H); ${ }^{13} \mathrm{C} \mathrm{NMR}\left(\mathrm{CDCl}_{3}\right) \delta 172.4,172.1$, $171.3,156.4,155.7,136.7,135.8,129.3,128.5,128.2,128.1,126.7,80.3,67.3,63.7,55.8,51.8$, 49.9, 38.3, 28.2, 17.5. Anal. Calcd for $\mathrm{C}_{28} \mathrm{H}_{35} \mathrm{~N}_{3} \mathrm{O}_{9}$ : C, 60.31; H, 6.33; N, 7.54; Found C, 60.05; H, 6.77; N, 7.39.

$\mathrm{N}$-(((Benzyloxy)carbonyl)-L-phenylalanyl)-O-((tert-butoxycarbonyl)glycyl)-L-threonine (Cbz-L-Phe-L-Thr(Boc-Gly)-OH, 4f). Yellow oil (80\%); ${ }^{1} \mathrm{H}$ NMR $\left(\mathrm{CDCl}_{3}\right) \delta$ 7.30-7.17 (m, $10 \mathrm{H}), 6.28$ (br s, 3H), 5.80-5.75 (m, 1H), 5.44-5.40 (m, 1H), 5.32-5.30 (m, 1H), 5.10-4.95 (m, $2 \mathrm{H}), 4.80-4.75(\mathrm{~m}, 1 \mathrm{H}), 4.70-4.54(\mathrm{~m}, 2 \mathrm{H}), 3.80-3.78(\mathrm{~m}, 2 \mathrm{H}), 3.15-3.13(\mathrm{~m}, 1 \mathrm{H}), 3.07-2.98$ $(\mathrm{m}, 1 \mathrm{H}), 2.04$ (br s, 2H), 1.41 (br s, 9H), 1.27-1.23 (m, 3H); ${ }^{13} \mathrm{C} \mathrm{NMR}\left(\mathrm{CDCl}_{3}\right) \delta 172.4,172.2$, $171.7,171.3,156.4,136.3,129.4,128.6,128.5,128.2,128.0,127.0,80.5,67.2,60.5,56.2$, 55.7, 42.3, 28.3, 17.1, 14.2. Anal. Calcd for $\mathrm{C}_{28} \mathrm{H}_{35} \mathrm{~N}_{3} \mathrm{O}_{9}$ : C, 59.04; H, 6.43; N, 7.38; Found C, 59.40; $\mathrm{H}, 6.76 ; \mathrm{N}, 7.00$.

O-(((Benzyloxy)carbonyl)-L-alanyl)- $N$-((tert-butoxycarbonyl)-L-phenylalanyl)-L-threonine (Boc-L-Phe-L-Thr(Cbz-L-Ala)-OH, 4g). White solid (83\%); mp 72-74 ${ }^{\circ} \mathrm{C} ;{ }^{1} \mathrm{H}$ NMR $\left(\mathrm{CDCl}_{3}\right) \delta$ 10.24 (s, 1H), 7.30-7.16 (m, 10H), 5.44 (dd, $J 6.4,3.8 \mathrm{~Hz}, 1 \mathrm{H}), 5.14-5.07$ (m, 2H), 4.80 (dd, $J$ 8.9, 3.5 Hz, 1H), 4.60-4.47(m, 1H), 4.32-4.24 (m, 1H), 3.23-2.94 (m, 2H), 1.43-1.23 (m, 15H); ${ }^{13} \mathrm{C} \mathrm{NMR}\left(\mathrm{CDCl}_{3}\right) \delta 173.1,173.0,171.8,156.5,156.3,136.9,136.3,129.6,128.7,128.3,127.0$, 80.5, 71.7, 67.4, 55.8, 49.9, 38.1, 28.4, 18.6, 17.9, 17.3. Anal. Calcd for $\mathrm{C}_{29} \mathrm{H}_{37} \mathrm{~N}_{3} \mathrm{O}_{9}$ : C, 60.93; H, 6.52; N, 7.35; Found C, 61.24; H, 6.64; N, 7.55.

\section{General procedure for the preparation of unprotected 0 -acyl isopeptides 5a-g}

For deprotection of the Cbz- protecting group. Compound $4 \mathbf{a}-\mathbf{d}$, $4 \mathrm{f}$ and $\mathbf{4 g}$ (1.0 mmol) was dissolved in anhydrous $\mathrm{MeOH}(30 \mathrm{~mL})$ and stirred under an atmosphere of hydrogen in the presence of a catalytic amount of $\mathrm{Pd} / \mathrm{C}$ for $4 \mathrm{~h}$. Filtration through a bed of celite and evaporation afforded 5a-d, 5f and 5g. Compound $\mathbf{4 f}$ was deprotected and used as crude to make compound 8b.

For deprotection of the Boc-protecting group. Compounds $4 \mathrm{e}$ and $4 \mathrm{~h}$ (1.0 mmol) was dissolved in either $\mathrm{HCl}$-dioxane $(4.0 \mathrm{M} \mathrm{HCl}$ in dioxane, $15 \mathrm{~mL}$ ) or freshly prepared $\mathrm{HCl}-\mathrm{MeOH}$ (prepared by bubbling $\mathrm{HCl}$ in $\mathrm{MeOH})(15 \mathrm{~mL})$ and stirred for $2 \mathrm{~h}$. Solvent is evaporated, and ether was added to the residue and stirred for $2 \mathrm{~h}$. Filtration gave a white solid $\mathbf{5 e}$ and $\mathbf{5 h}$ (when sticky solid resulted, decantation of ether several times was performed instead).

$\mathbf{N}$-(L-Phenylalanyl)-O-((tert-butoxycarbonyl)glycyl)-L-serine (L-Phe-L-Ser(Boc-Gly)-OH 5a). White solid (80\%); mp 170-172 ${ }^{\circ} \mathrm{C} ;{ }^{1} \mathrm{H}$ NMR ( $\left.\mathrm{CD}_{3} \mathrm{OD}\right) \delta 7.35-7.24(\mathrm{~m}, 5 \mathrm{H}), 4.60-4.50(\mathrm{~m}$, $1 \mathrm{H}), 4.39(\mathrm{~s}, 2 \mathrm{H}), 4.25-4.18(\mathrm{~m}, 1 \mathrm{H}), 3.77(\mathrm{~s}, 2 \mathrm{H}), 3.30-3.00(\mathrm{~m}, 2 \mathrm{H}), 1.38(\mathrm{~s}, 9 \mathrm{H}) ;{ }^{13} \mathrm{C} \mathrm{NMR}$ $\left(\mathrm{CD}_{3} \mathrm{OD}\right) \delta 174.2,172.8,169.5,158.8,135.5,130.7,130.3,129.0,81.5,66.5,55.9,55.6,43.1$, 38.2, 28.8. HRMS $m / z$ for $\mathrm{C}_{19} \mathrm{H}_{28} \mathrm{~N}_{3} \mathrm{O}_{7}[\mathrm{M}+\mathrm{H}]^{+}$calcd. 410.1922, found 410.1909. 
$\mathrm{N}$-(L-Alanyl)-O-((tert-butoxycarbonyl)-L-phenylalanyl)-L-serine (L-Ala-L-Ser(Boc-L-Phe)OH, 5b). White solid (79\%); mp $150-152{ }^{\circ} \mathrm{C}$; ${ }^{1} \mathrm{H}$ NMR $\left(\mathrm{CD}_{3} \mathrm{OD}\right) \delta 7.28-7.17$ (m, 5H), 4.53$4.33(\mathrm{~m}, 4 \mathrm{H}), 3.18$ (dd, $J 13.9,4.7 \mathrm{~Hz}, 1 \mathrm{H}), 2.87$ (dd, $J 13.9,9.5 \mathrm{~Hz}, 1 \mathrm{H}), 1.53(\mathrm{~d}, J 6.7 \mathrm{~Hz}, 3 \mathrm{H})$, $1.36(\mathrm{~s}, 9 \mathrm{H}) ;{ }^{13} \mathrm{C}$ NMR $\left(\mathrm{CD}_{3} \mathrm{OD}\right) \delta 174.2,173.4,170.8,157.8,138.5,130.5,130.3,129.4,127.7$, 80.6, 66.5, 56.5, 55.6, 50.4, 38.4, 28.7, 17.5. Anal. Calcd for $\mathrm{C}_{20} \mathrm{H}_{29} \mathrm{~N}_{3} \mathrm{O}_{7}$ : C, 56.73; H, 6.90; N, 9.92; Found C, 56.61; H, 7.33; N, 9.18 .

$\mathrm{N}$-(L-Alanyl)-O-((tert-butoxycarbonyl)glycyl)-L-serine (L-Ala-L-Ser(Boc-Gly)-OH, 5c). White solid (85\%); mp; 108-110; ${ }^{1} \mathrm{H}$ NMR (CD $\left.\mathrm{OD}\right) \delta 4.42-4.30(\mathrm{~m}, 2 \mathrm{H}), 3.85-3.83(\mathrm{~m}, 1 \mathrm{H})$, 3.63 (br s, 2H), 3.20-3.14 (m, 1H), $1.38(\mathrm{~d}, J 7.2 \mathrm{~Hz}, 3 \mathrm{H}), 1.29(\mathrm{~s}, 9 \mathrm{H}) ;{ }^{13} \mathrm{C} \mathrm{NMR}\left(\mathrm{CD}_{3} \mathrm{OD}\right) \delta$ 174.5, 172.3, 171.0, 158.7, 80.8, 66.5, 55.7, 43.1, 28.9, 17.7. Anal. Calcd for $\mathrm{C}_{13} \mathrm{H}_{23} \mathrm{~N}_{3} \mathrm{O}_{7}$ : C, 46.84; H, 6.95; N, 12.61; Found C, 46.36; H, 7.34; N, 12.08 .

$\mathbf{N}$-(L-Alanyl)-O-((tert-butoxycarbonyl)-L-valyl)-L-serine (L-Ala-L-Ser(Boc-L-Val)-OH, 5d). Gum (75\%); ${ }^{1} \mathrm{H}$ NMR (CD $\left.{ }_{3} \mathrm{OD}\right) \delta 4.67-4.38(\mathrm{~m}, 3 \mathrm{H}), 4.10-3.28(\mathrm{~m}, 2 \mathrm{H}), 2.21-2.03(\mathrm{~m}, 1 \mathrm{H})$, $1.56(\mathrm{~d}, J 6.1 \mathrm{~Hz}, 3 \mathrm{H}), 1.44(\mathrm{~s}, 9 \mathrm{H}), 0.92(\mathrm{dd}, J 11.3,6.8 \mathrm{~Hz}, 6 \mathrm{H}) ;{ }^{13} \mathrm{C} \mathrm{NMR}\left(\mathrm{CD}_{3} \mathrm{OD}\right) \delta 173.6$, $170.8,158.4,80.7,66.2,60.7,50.5,42.2,31.8,28.9$, 19.8, 18.5. HRMS $m / z$ for $\mathrm{C}_{16} \mathrm{H}_{29} \mathrm{~N}_{3} \mathrm{O}_{7}$ $[\mathrm{M}+\mathrm{H}]^{+}$calcd. 376.2010, found 376.2039.

$\mathrm{N}$-(L-Phenylalanyl)-O-(((benzyloxy)carbonyl)-L-alanyl)-L-serine (L-Phe-L-Ser(Cbz-L-Ala)OH, 5e). White microcrystals (95\%); mp 103-104 ${ }^{\circ} \mathrm{C}$; ${ }^{1} \mathrm{H}$ NMR (DMSO- $\left.d_{6}\right) \delta 9.15$ (d, $J 8.1 \mathrm{~Hz}$, $1 \mathrm{H}), 8.37$ (br s, 3H), 7.82 (d, J 7.2 Hz, 1H), 7.39-7.23 (m, 10H), 4.99 (dd, J 15.7, $12.6 \mathrm{~Hz}, 2 \mathrm{H})$, 4.65-4.59 (m, 1H), 4.36 (dd, J 11.3, 4.7 Hz, 1H), 4.27 (dd, $J 11.3,5.9 \mathrm{~Hz}, 1 \mathrm{H}), 4.16-4.05$ (m, 2H), $3.20(\mathrm{dd}, J 14.3,5.7 \mathrm{~Hz}, 1 \mathrm{H}), 3.03(\mathrm{dd}, J 14.3,7.5 \mathrm{~Hz}), 1.29$ (d, $J 7.4 \mathrm{~Hz}, 3 \mathrm{H}) ;{ }^{13} \mathrm{C}$ NMR $\left(\mathrm{DMSO}-d_{6}\right) \delta 172.6,170.0,168.2,155.9,136.9,134.8,129.7,128.5,128.4,127.8,127.1,66.4$, 65.6, 53.2, 51.2, 49.3, 36.7, 16.9. Anal. Calcd for $\mathrm{C}_{46} \mathrm{H}_{58} \mathrm{~N}_{6} \mathrm{O}_{15}: \mathrm{C}, 54.93 ; \mathrm{H}, 5.81$; N, 8.35; Found C, 54.63; H, 6.27; N, 8.02.

$\mathrm{N}$-(L-Phenylalanyl)-O-((tert-butoxycarbonyl)glycyl)-L-threonine $\quad$ (L-Phe-L-Thr(Boc-Gly)$\mathbf{O H}, \mathbf{5 f})$. Used as crude to make compound $\mathbf{8 b}$. See $\mathbf{8 b}$.

$\mathrm{N}$-(L-Phenylalanyl)-O-(((benzyloxy)carbonyl)-L-alanyl)-L-threonine (L-Phe-L-Thr(Cbz-LAla)-OH, 5g). White solid (92\%); mp 93-95 ${ }^{\circ} \mathrm{C} ;{ }^{1} \mathrm{H}$ NMR $\left(\mathrm{CD}_{3} \mathrm{OD}\right) \delta 7.35-7.28(\mathrm{~m}, 10 \mathrm{H})$, 5.46-5.38 (m, 1H), 5.07 (s, 2H), 4.70 (d, J 5.0 Hz, 1H), 4.30 (dd, J 8.3, 5.7 Hz, 1H), 4.234.15(m, 1H), 3.34 (dd, $J$ 9.8, $4.4 \mathrm{~Hz}, 1 \mathrm{H}), 3.08$ (dd, $J 14.3,8.4 \mathrm{~Hz}, 1 \mathrm{H}), 1.37$ (d, $J 7.3 \mathrm{~Hz}, 3 \mathrm{H})$, $1.31(\mathrm{~d}, J 6.4 \mathrm{~Hz}, 3 \mathrm{H}) ;{ }^{13} \mathrm{C} \mathrm{NMR}\left(\mathrm{CD}_{3} \mathrm{OD}\right) \delta 173.8,171.6,170.3,158.6,138.2,135.5,130.8$, $130.2,129.6,129.2,129.0,128.8,72.0,67.8,57.5,55.7,51.2,38.6,17.6,17.5$. Anal. Calcd for $\mathrm{C}_{48} \mathrm{H}_{62} \mathrm{Cl}_{2} \mathrm{~N}_{6} \mathrm{O}_{15}:$ C, 55.76; H, 6.04; N, 8.13; Found C, 55.59; H, 6.35; N, 7.98.

General procedure for the preparation of unprotected $O$-acyl isopeptides 8a-c Compounds $8 \mathbf{a}-\mathbf{c}$ were prepared by following the same procedure followed for $4 \mathbf{a}-\mathbf{g}$. $\mathrm{N}$-((Benzyloxy)carbonyl)glycyl-L-phenylalanyl-O-((tert-butoxycarbonyl)glycyl)-L-serine (Cbz-Gly-L-Phe-L-Ser(Boc-Gly)-OH, 8a). White solid (89\%), converted to compound 9a after checking NMR. 8a: mp $180-181{ }^{\circ} \mathrm{C}$ (decomposed). ${ }^{1} \mathrm{H}$ NMR $\left(\mathrm{CD}_{3} \mathrm{OD}\right) \delta 7.39-7.22(\mathrm{~m}, 10 \mathrm{H})$, 5.08 (s, 2H), 4.75-4.63 (m, 2H), 4.57-4.49 (m, 1H), 4.43-4.37 (m, 1H), 3.79-3.66 (m, 4H), 
3.30-3.06 (m, 1H), 2.97-2.77 (m, 4H), $1.42(\mathrm{~s}, 9 \mathrm{H}) ;{ }^{13} \mathrm{C}$ NMR $\left(\mathrm{CD}_{3} \mathrm{OD}\right) \delta 177.0,173.6,173.4$, $172.1,171.9,171.8,159.1,138.3,130.5,129.6,129.1,129.0,127.9,127.2,80.9,74.3,68.0,65.0$, 55.7, 53.0, 45.0, 44.0, 43.0, 38.7, 28.6. HRMS $m / z$ for $\mathrm{C}_{29} \mathrm{H}_{36} \mathrm{~N}_{4} \mathrm{O}_{10}[\mathrm{M}+\mathrm{H}]^{+}$calcd. 601.2441, found 601.2402 .

$\mathrm{N}$-((Benzyloxy)carbonyl)glycyl-L-alanyl-O-((tert-butoxycarbonyl)-L-phenylalanyl)-L-serine (Cbz-Gly-L-Ala-L-Ser(Boc-L-Phe)-OH, 8b). Colorless oil (89\%); converted to compound 9b after checking NMR. 8b: ${ }^{1} \mathrm{H}$ NMR $\left(\mathrm{CDCl}_{3}\right) \delta 9.70$ (br s, 2H), 7.34 (d, J 7.4 Hz, 1H), 7.25-7.07 $(\mathrm{m}, 10 \mathrm{H}), 6.03(\mathrm{~d}, J 7.4 \mathrm{~Hz}, 1 \mathrm{H}), 5.07-4.57(\mathrm{~m}, 4 \mathrm{H}), 4.29-4.14(\mathrm{~m}, 2 \mathrm{H}), 3.84-3.55(\mathrm{~m}, 3 \mathrm{H})$, 3.12-2.89 (m, 2H), $1.36(\mathrm{~d}, J 2.8 \mathrm{~Hz}, 3 \mathrm{H}), 1.32(\mathrm{~s}, 9 \mathrm{H}) ;{ }^{13} \mathrm{C} \mathrm{NMR}\left(\mathrm{CDCl}_{3}\right) \delta 171.5,170.3,170.0$, $169.4,155.5,155.1,135.1,134.9,128.2,127.5,127.4,127.1,127.0,125.9,124.4,66.1,62.6$, 53.0, 50.9, 48.8, 42.8, 36.5, 29.3, 27.2, 16.4. HRMS $m / z$ for $\mathrm{C}_{30} \mathrm{H}_{38} \mathrm{~N}_{4} \mathrm{O}_{10}[\mathrm{M}+\mathrm{H}]^{+}$calcd. 615.2584, found 6159.2601.

$\mathrm{N}$-((Benzyloxy)carbonyl)glycyl-L-phenylalanyl-O-((tert-butoxycarbonyl)glycyl)-L-threonine (Cbz-Gly-L-Phe-L-Thr(Boc-Gly)-OH, 8c). Colorless oil (82\%); ${ }^{1} \mathrm{H}$ NMR $\left(\mathrm{CD}_{3} \mathrm{OD}\right) \delta 7.37-7.20$ (m, 10H), 5.10-5.08 (m, 2H), 4.13-4.08 (m, 1H), 3.83-3.29 (m, 3H), 2.01-1.97 (m, 8H), 1.44 (br $\mathrm{s}, 9 \mathrm{H}), 1.23(\mathrm{t}, J 7.2 \mathrm{~Hz}, 3 \mathrm{H}) ;{ }^{13} \mathrm{C} \mathrm{NMR}\left(\mathrm{CD}_{3} \mathrm{OD}\right) \delta 175.4,174.5,174.0,173.8,173.2,138.3$, $130.5,129.6,129.2,129.1,129.0,129.0,128.0,80.7,68.0,67.8,61.7,55.1,45.0,43.3,42.9$, 38.9, 38.5, 28.8, 21.0, 20.9, 14.6. HRMS $m / z$ for $\mathrm{C}_{21} \mathrm{H}_{30} \mathrm{~N}_{4} \mathrm{O}_{8} \mathrm{Na}[\mathrm{M}+\mathrm{Na}]^{+}$calcd. 489.1956, found 489.1965. HRMS $m / z$ for $\mathrm{C}_{30} \mathrm{H}_{38} \mathrm{~N}_{4} \mathrm{O}_{10}[\mathrm{M}+\mathrm{H}]^{+}$calcd. 615.2584, found 6159.2596.

General procedure for the preparation of unprotected 0 -acyl isopeptides 9a,b Compounds 9a,b were prepared by following the same procedure followed for $\mathbf{5 a}-\mathbf{g}$.

O-((tert-Butoxycarbonyl)glycyl)- $\boldsymbol{N}$-glycyl-L-phenylalanyl-L-serine (Gly-L-Phe-L-Ser(BocGly)-OH, 9a). White solid (85\%) yield; mp $168-173{ }^{\circ} \mathrm{C} ;{ }^{1} \mathrm{H}$ NMR $\left(\mathrm{CD}_{3} \mathrm{OD}\right) \delta$ 7.41-7.11 (m, $5 \mathrm{H}), 4.78-4.63(\mathrm{~m}, 1 \mathrm{H}), 4.62-4.49(\mathrm{~m}, 2 \mathrm{H}), 4.43-4.33(\mathrm{~m}, 1 \mathrm{H}), 3.93-3.63(\mathrm{~m}, 3 \mathrm{H}), 3.27-3.18$ $(\mathrm{m}, 1 \mathrm{H}), 3.01-2.63(\mathrm{~m}, 4 \mathrm{H}), 1.43(\mathrm{~s}, 9 \mathrm{H}) ;{ }^{13} \mathrm{C} \mathrm{NMR}\left(\mathrm{CD}_{3} \mathrm{OD}\right) \delta 174.9,173.2,172.2,167.8$, $158.7,138.5,130.5,129.7,128.0,80.9,66.0,56.6,54.7,44.8,43.1,38.8,28.9$. HRMS $m / z$ for $\mathrm{C}_{21} \mathrm{H}_{30} \mathrm{~N}_{4} \mathrm{O}_{8} \mathrm{Na}[\mathrm{M}+\mathrm{Na}]^{+}$calcd. 489.1956, found 489.1965.

$\boldsymbol{O}$-((tert-Butoxycarbonyl)glycyl)- $\boldsymbol{N}$-glycyl-L-phenylalanyl-L-threonine

(Gly-L-Phe-LThr(Boc-Gly)-OH, 9b). Sticky yellow solid (75\%); ${ }^{1} \mathrm{H}$ NMR $\left(\mathrm{CDCl}_{3}\right) \delta 7.30-7.13(\mathrm{~m}, 5 \mathrm{H})$, 5.14-4.49 (m, 1H), 3.75-3.70 (m, 2H), 3.10-3.06 (m, 1H), 1.80-1.50 (m, 3H), 1.45-1.10 (m, $12 \mathrm{H}) ;{ }^{13} \mathrm{C} \mathrm{NMR}\left(\mathrm{CDCl}_{3}\right) \delta 171.7,170.1,168.5,152.0,151.4,132.0,125.5,125.3,124.6,123.1$, 76.3, 52.0, 50.5, 50.3, 48.3, 38.3, 34.4, 33.9, 29.6, 24.3, 22.2. HRMS $m / z$ for $\mathrm{C}_{22} \mathrm{H}_{32} \mathrm{~N}_{4} \mathrm{O}_{8}$ $[\mathrm{M}+\mathrm{H}]^{+}$calcd. 481.2320, found 481.2334.

(tert-Butoxycarbonyl)glycyl-L-phenylalanyl-L-serine (Boc-Gly-L-Phe-L-Ser-OH, 6a). Compound 5a (20 mg, $0.05 \mathrm{mmol})$ was dissolved in piperidine $20 \mathrm{v} / \mathrm{v} \%$ in DMF $(1 \mathrm{~mL})$ and stirred at $50{ }^{\circ} \mathrm{C}$ and $50 \mathrm{~W}$ for $1 \mathrm{~h}$. The mixture was then evaporated and purified by HPLC to give ligated product 6a (57\%); The sample was analyzed via reverse phase gradient C18 HPLC/UV/()ESI-MSn to give a retention time of $23.07 \mathrm{~min}$. To confirm structure, HRMS for $\mathbf{6 a} \mathrm{m} / \mathrm{z}$ for $\mathrm{C}_{19} \mathrm{H}_{26} \mathrm{~N}_{3} \mathrm{O}_{7}[\mathrm{M}-\mathrm{H}]^{+}$calcd. 408.1776, found 408.1794. 
(tert-Butoxycarbonyl)glycylglycyl-L-phenylalanyl-L-serine (Boc-Gly-Gly-L-Phe-L-Ser-OH, 10a). Compound 9a (20 mg, $0.04 \mathrm{mmol})$ was dissolved in piperidine $20 \mathrm{v} / \mathrm{v} \%$ in DMF ( $1 \mathrm{~mL})$ and stirred at $50{ }^{\circ} \mathrm{C}$ and $50 \mathrm{~W}$ for $1 \mathrm{~h}$ ( $3 \mathrm{~h}$ for $9 \mathrm{~b}$ ). The mixture was then evaporated and purified by HPLC to give ligated product 10a $(99.39 \%)$. The sample was analyzed via reverse phase gradient C18 HPLC/UV (254 nm/ESI-MSn gave a retention time of $21.67 \mathrm{~min}$ (for 10a). To confirm structure, HRMS for 11a $m / z$ for $\mathrm{C}_{21} \mathrm{H}_{29} \mathrm{~N}_{4} \mathrm{O}_{8}[\mathrm{M}-\mathrm{H}]^{+}$calcd. 465.2064, found 465.1992.

\section{Acknowledgements}

We thank the University of Florida and the Kenan Foundation for financial support. This paper was also funded by the Deanship of Scientific Research (DSR), King Abdulaziz University, Jeddah, under Grant no. (24-3-1432/HiCi). The authors, therefore, acknowledge with thanks DSR technical and financial support. The authors, therefore, acknowledge the technical and financial support of KAU. We also thank to Dr. C. D. Hall for helpful suggestions.

\section{References}

1. Fjell, C. D.; Hiss, J. A.; Hancock, R. E. W.; Schneider, G. Nat. Rev. Drug Discov. 2012, 11, 37-51.

2. Castanho, M.; Santos, N. C. Peptide Drug Discovery and Development: Translational Research in Academia and Industry, $1^{\text {st }}$ edn., Wiley-VCH Verlag GmbH \& Co. KGaA, Weinheim, 2011.

3. Groner, B. Peptides as Drugs. Discovery and Development, Wiley-VCH Verlag GmbH \& Co. KGaA, Weinheim, 2009.

4. White, C. J.; Yudin, A. K. Nat. Chem. 2011, 3, 509-524. http://dx.doi.org/10.1038/nchem.1062

5. 5. Dawson, P. E.; Muir, T. W.; Clark-Lewis, I.; Kent, S. B. H. Science 1994, 266, 776-779. http://dx.doi.org/10.1126/science.7973629

6. Kent, S. B. H. Chem. Soc. Rev. 2009, 38, 338-351. http://dx.doi.org/10.1039/b700141j

7. Yeo, D.S. Y.; Srinivasan, R.; Chen, G. Y. J.; Yao, S. Q. Chem. Eur. J. 2004, 10, 4664-4672. http://dx.doi.org/10.1002/chem.200400414

8. Wieland, T.; Bokelmann, E.; Bauer, L.; Lang, H. U.; Lau, H.; Schafer, W. Justus Liebigs Ann. Chem. 1953, 583, 129-149.

http://dx.doi.org/10.1002/jlac.19535830110

9. Merrifield, B. Science 1986, 232, 341-347.

http://dx.doi.org/10.1126/science.3961484

10. Heapy, A. M.; Williams, G. M.; Fraser, J. D.; Brimble, M. A. Org. Lett. 2012, 14, 878-881. 
http://dx.doi.org/10.1021/o1203407z

11. Sakamoto, I.; Tezuka, K.; Fukae, K.; Ishii, K.; Taduru, K.; Maeda, M.; Ouchi, M.; Yoshida, K.; Nambu, Y.; Igarashi, J.; Hayashi, N.; Tsuji, T.; Kajihara, Y. J. Am. Chem. Soc. 2012, $134,5428-5431$.

http://dx.doi.org/10.1021/ja2109079

12. Mandal, K.; Kent, S. B. H. Angew. Chem., Int. Ed. 2011, 50, 8029-8033.

http://dx.doi.org/10.1002/anie.201103237

13. Luisier, S.; Avital-Shmilovici, M.; Weiss, M. A.; Kent, S. B. H. Chem. Comm. 2010, 46, 8177-8179.

http://dx.doi.org/10.1039/c0cc03141k

14. Li, X.; Lam, H. Yung; Z., Yinfeng; C., Chun K. Org. Lett. 2010, 12, 1724-1727. http://dx.doi.org/10.1021/ol1003109

15. Hackenberger, C. P. R.; Schwarzer, D. Angew. Chem., Int. Ed. 2008, 47, 10030-10074. http://dx.doi.org/10.1002/anie.200801313

16. Dirksen, A.; Dawson, P. E. Curr. Opin. Chem. Biol. 2008, 12, 760-766. http://dx.doi.org/10.1016/j.cbpa.2008.10.009

17. Johnson, E. C. B.; Kent, S. B. H. J. Am. Chem. Soc. 2006, 128, 6640-6646. http://dx.doi.org/10.1021/ja058344i

18. Bacsa, B.; Bősze, S.; Kappe, C. O. J. Org. Chem. 2010, 75, 2103-2106. http://dx.doi.org/10.1021/jo100136r

19. Sohma, Y.; Yoshiya, T.; Taniguchi, A.; Kimura, T.; Hayashi, Y.; Kiso, Y. Biopolymers 2007, $88,253-262$.

http://dx.doi.org/10.1002/bip.20683

20. El Khatib, M.; Jauregui, L.; Tala, S. R.; Khelashvili, L.; Katritzky, A. R. MedChemComm 2011, 2, 1087-1092. http://dx.doi.org/10.1039/c1md00130b

21. Katritzky, A. R.; Tala, S. R.; Abo-Dya, N. E.; Ibrahim, T. S.; El-Feky, S. A.; Gyanda, K.; Pandya, K. M. J. Org. Chem. 2011, 76, 85-96. http://dx.doi.org/10.1021/jo1015757

22. Ha, K.; Chahar, M.; Monbaliu, J.-C. M.; Todadze, E.; Hansen, F. K.; Oliferenko, A. A.; Ocampo, C. E.; Leino, D.; Lillicotch, A.; Stevens, C. V.; Katritzky, A. R. J. Org. Chem. 2012, 77, 2637-2648. http://dx.doi.org/10.1021/jo2023125

23. Panda, S. S.; El-Nachef, C.; Bajaj, K.; Al-Youbi, A. O.; Oliferenko, A.; Katritzky, A. R. Chem. Biol. Drug Des. 2012, 80, 821-827. http://dx.doi.org/10.1111/cbdd.12053

24. Popov, V.; Panda, S. S.; Katritzky, A. R. Org. Biomol. Chem. 2013, 11, 1594-1597. http://dx.doi.org/10.1039/c3ob27421g

25. Popov, V.; Panda, S. S.; Katritzky, A. R. J. Org. Chem. 2013, 78, 7455-7461. http://dx.doi.org/10.1021/jo4009468 
26. El Khatib, M.; Elagawany, M.; Jabeen, F.; Todadze, E.; Bol'shakov, O.; Oliferenko, A.; Khelashvili, L.; El-Feky, S. A.; Asiri, A.; Katritzky, A. R. Org. Biomol. Chem. 2012, 10, 4836-4838.

http://dx.doi.org/10.1039/c2ob07050b

27. Oliferenko, A. A.; Katritzky, A. R. Org. Biomol. Chem. 2011, 9, 4756-4759. http://dx.doi.org/10.1039/c1ob05536d

28. Katritzky, A. R.; Shestopalov, A. A.; Suzuki, K. Synthesis 2004, 1806-1813. http://dx.doi.org/10.1055/s-2004-829126

29. Meneses, C.; Nicoll, S. L.; Trembleau, L. J. Org. Chem. 2010, 75, 564-569. http://dx.doi.org/10.1021/jo902116p 